\title{
COALESCENCIA: UNA APROXIMACIÓN A LA ESCRITURA DE HÉLĖNE CIXOUS
}

\author{
GERARDO RODRÍGUEZ SALAS \\ Universidad de Granada
}

\section{INTRODUCCIÓN}

Para entender la producción crítica y literaria de Hélène Cixous, hemos de relacionar su trabajo con el de Jacques Derrida, puesto que la teoría crítica de Cixous que denominaremos "coalescencia» es una clara aplicación al ámbito de la diferencia sexual del método desconstructivista derridiano. Al explicar la desconstrucción, Derrida propone:

"a double writing, that is, a writing that is in and of itself multiple, what I called, in 'La double séance', a double science». ${ }^{1}$

Distingue, por tanto, dos fases dentro de este movimiento que son necesarias para trascender el sistema dominante de oposiciones binarias: una primera acción desconstructiva, o inversión jerárquica, que debe ser superada por una «subversión» que conduzca a un modelo radicalmente distinto. Derrida clarifica este segundo paso afirmando que:

"we must also mark the interval between inversion, which brings low what was high, and the irruptive emergence of a new 'concept', a concept that can no longer be, and never could be, included in the previous regime». ${ }^{2}$

Este capítulo analiza la forma en que Cixous se adhiere al modelo desconstructivo en su propuesta de lo que sus traductores ingleses denominan «blending». Importantes críticas como Verena Andermatt Conley o Susan Sellers usan este término para referirse a la escritura de Cixous. Sin embargo, el presente estudio propone "coalescencia» como un término alternativo para describir la filosofía de esta crítica y escritora francesa a partir del verbo «coalesce». Ofrecemos, por tanto, una definición de este término, extraída del

1. DerRIDA, Jacques: Positions (Trad. Allan Bass), Londres, The Athlone Press, 1987 (1972), p. 41.

2. Ibíd., p. 42. 
Webster Dictionary (2003): «(2) to unite or merge into one body or mass; (3) to unite, combine, or blend into a single body or group, as individuals, parties or nations». Lo importante de esta definición es que, en comunión con las teorías de Cixous, aunque se habla de la combinación de distintos componentes, en la "coalescencia», o formación de un todo, cada uno de estos términos retiene su propia idiosincrasia.

Una vez aclarados sus presupuestos teóricos, se aplicarán a su narrativa experimental Angst, originalmente publicada en Francia en 1977. Hemos seleccionado este trabajo porque, aunque esta crítica y escritora ha sido ampliamente estudiada, este texto en particular ha recibido escasa atención crítica. De este modo, ofrecemos una nueva aproximación a esta obra, al igual que a la teoría crítica de Cixous a lo largo del presente estudio.

\section{CIXOUS Y SU CONTEXTO}

Para entender el uso estratégico que Cixous hace de su "coalescencia», es necesario ubicarla dentro del contexto del denominado Psych et Po (i.e. Psychanalyse et Politique), un grupo feminista francés de finales de los setenta del que Cixous formó parte durante un tiempo y del que derivó muchas de las ideas revolucionarias que discutiremos. Como Cixous, Antoinette Fouque -la líder carismática del Psych et Po- propone el término "alterity» para definir a la mujer, que conlleva una diferencia radical frente a una diferencia relativa con respecto al hombre. ${ }^{3}$ Además, siguiendo las ideas de este grupo, Cixous ofrece una definición alternativa de la noción de la sexualidad femenina, de tal modo que no depende necesariamente del hombre, y celebra el cuerpo de la mujer, que representa el eje central sobre el que gira su narrativa. Igualmente, se hace eco de la batalla del Psych et Po con el mecanismo del lenguaje, cuestionando tanto su sintaxis como su significado. Esta rebeldía hacia el lenguaje se plasma en su narrativa, puesto que desdeña el carácter restrictivo de esta herramienta lingüística para ofrecer una visión radicalmente polisémica que entronca con la complejidad de la subjetividad femenina que se esfuerza en crear.

Antoinette Fouque clasifica el Psych et Po como "post-feminista", afirmando que:

«[w]e are neither pre- nor antifeminist but post-feminist; we work for heterosexuality.... Contrary to what most people believe, our enemy isn't man but phallocracy; that is, the imperialism of the phallus». ${ }^{4}$

Ciertamente, como postulamos más adelante, en el modelo teórico de Cixous existe un lugar para los hombres que son capaces de comulgar con su concepto de multiplicidad. En relación con esta multiplicidad, también mencionamos la empresa cultural polivalente de esta autora. Cixous es una crítica de inspiración psicoanalítica y semiótica que defendió los derechos de las mujeres

3. Cf. en DUCHEN, Claire (ed. y trad): Explorations in Feminism. French Connections: Voices from the Women's Movement in France, Londres, Hutchinson, 1987, p. 49.

4. Cf. en Ibíd., p. 52. 
en el contexto de la novela experimental. Es precisamente este contexto el que le permite investigar el área indeterminada entre el discurso crítico y ficcional, en cuya conexión encontramos el epítome de su modelo de "coalescencia». Sin embargo, su pluralismo cultural también deriva de su propia experiencia personal, que le permitió interactuar con tres grandes culturas: árabe (nació en Argelia en 1937), francesa (a través de su padre) y alemana (a través de su madre). Este multiculturalismo explica su defensa del oprimido (no sólo de las mujeres), puesto que, tras establecerse en Francia, se vio a sí misma como una "proscrita» social, perteneciendo al menos a tres grupos desfavorecidos: mujeres, extranjeros y lesbianas.

\section{ANGST}

En su ficción Angst, Cixous parte de una premisa: la protagonista es una mujer. Aunque este personaje presenta una identidad compleja, incluso caótica a lo largo de toda la narrativa, las alusiones a su cuerpo de mujer son frecuentes. La pista más significativa es el epitafio que da comienzo al libro, que Cixous dedica "to the Vital woman, to whom this text did not know it would lead». ${ }^{5}$ El objetivo de Cixous radica en exponer la opresión de las mujeres dentro del orden simbólico dominante del patriarcado. Sin embargo, a pesar de escoger a una mujer como la figura central de su narrativa, propone un ámbito alternativo donde tanto los valores femeninos como los masculinos podrían coexistir. Su compromiso con la coalescencia hace que Cixous presente a un sujeto indeterminado en el curso de su narrativa, a pesar de que sea biológicamente una mujer, de tal forma que no excluye en ningún momento la "masculinidad» de este nuevo proyecto. Como novela experimental, Angst carece de argumento en el sentido tradicional. El público lector accede a la confusión psicológica de una mujer (lo que refleja las influencias psicoanalistas de la autora) en su búsqueda de una identidad femenina distintiva que, en el curso del relato, demuestra ser, no univalente como el sistema patriarcal dicta, sino ilimitadamente compleja, conectando de este modo con los deseos indefinibles y reprimidos de las mujeres.

\section{INVERSIÓN DE OPOSICIONES BINARIAS}

El concepto de «blending", redesignado como "coalescencia», emerge cuando Cixous ataca la noción de oposiciones binarias que, en su opinión, y siguiendo los principios derridianos, constituye la base del orden patriarcal. En este modelo imperante todos los componentes se organizan en parejas de elementos opuestos, siendo uno de los miembros de la pareja «superior» al otro. Éste es el caso de la categoría "diferencia sexual», en la que Cixous se muestra especialmente interesada. Haciendo referencia al sistema patriarcal, alega:

5. Cixous, Hélène: Angst (Trad. Jo Levy), Londres, John Calder, 1985 (1977), p. 5. 
«things get hierarchical. Organization by hierarchy makes all conceptual organization subject to man. Male privilege, shown in the opposition between activity and passivity, which he uses to sustain himself». ${ }^{6}$

Sin embargo, resulta significativo descubrir que, aunque Cixous se refiere con frecuencia a las mujeres como la parte excluida en este cosmos binario, éstas no son "tachadas» del sistema dominante: conservan su existencia, pero permanecen dentro del sistema binario como el término oprimido. Ésta es la razón por la que, en Angst, Cixous elige retratar a una mujer como el reflejo de los valores femeninos "secundarios» del binarismo patriarcal, con el fin de subvertir esta visión a través de su proceso de coalescencia. De este modo, la protagonista revela su exclusión del modelo prevaleciente falocéntrico cuando afirma: "I felt myself summoned by the sentence that rejected me». ${ }^{7}$ A través de una sinécdoque, "the sentence» (una parte) representa al sistema completo (la lengua de una sociedad). Este sistema, según Cixous, ha estado dominado por el hombre desde sus comienzos y, por tanto, nunca ha ofrecido una representación adecuada de la mujer dentro del mismo.

Sue Thomas ilustra esta dualidad cuando concluye que:

"The stereotype (in Western thought) is structured by binary oppositions; the other of a dominant group is generally produced in stereotypical representation as the embodiment of negativity, the repository of negative attributes the dominant repress from their normative self-images».8

Esta idea fue inicialmente propuesta por Simone de Beauvoir, siendo más tarde adoptada por la propia Cixous cuando, en Angst, presenta a la protagonista como la parte negativa, según el punto de vista del patriarcado: "She has become the spirit of negation. What absence!». ${ }^{9}$ De este modo, la represión de la mujer no es más que el resultado del sistema patriarcal; no hay un lugar para ella en el orden simbólico. Cixous emplea una imagen muy efectiva para ilustrar la desubicación de la mujer en este sistema imperante: "the space is too narrow, your woman's hips are too wide, you are disturbed». ${ }^{10}$ Del mismo modo, resulta efectivo el carácter metafórico con que describe el proceso de la opresión femenina por el hombre. La multiplicidad inicial y riqueza interior de la mujer (representadas por el pavo real y su colorido) son reprimidas por el autoritario modelo falocéntrico y reemplazadas por una identidad univalente que no define a la mujer, pues no es más que una imitación de la identidad masculina. Siguiendo el estilo metafórico, esta identidad "gris» está representada por el gusano en el que se convierte el pavo real. ${ }^{11}$

6. CiXOus, Hélène y Catherine ClÉMENT: «Sorties: Out and Out: Attacks/Ways Out/Forays», en The Newly Born Woman (Trad. Betsy Wing), Londres, I.B. Tauris, 1996, p. 64.

7. Cixous, Hélène: Angst, op. cit, p. 135.

8. THOmAS, Sue: «Difference, Intersubjectivity, and Agency in the Colonial and Decolonizing Spaces of Hélène Cixous's 'Sorties'», Hypatia, 9:1 (1994), p. 55.

9. CiXous, Hélène: Angst, op. cit, p. 170.

10. Ibíd., p. 49.

11. Ibíd., pp. 49, 55. 
La limitación femenina se observa también en el retrato de la madre reprimida. Tradicionalmente, la maternidad se ha concebido como la cualidad femenina más distintiva, puesto que este atributo biológico diferencia a las mujeres de los hombres. Así, el nacimiento triunfante de un bebé es sustituido por un aborto monstruoso en la obra de Cixous, como una clara indicación de la forma en que el patriarcado priva a la feminidad de sus valores positivos y la convierte en algo negativo:

"The belly of the night is swollen... when things begin badly they don't ripen, they rot. The birth is a nightmare... no child, a monster comes out, it's a Mistake».12

La selección léxica en este pasaje indica la negatividad atribuida a la mujer y a su papel distintivo (en este caso, la maternidad y el embarazo). Incluso la figura de la madre se puede considerar tanto destructiva como compasiva, como un "tercer elemento» que logra establecer la armonía entre los términos opuestos del sistema binario. Sin embargo, mientras se encuentra inmersa en el sistema patriarcal, la protagonista es incapaz de articular este simbólico número tres: "One! Two! Mother says: One! Life says: Two! But when I wanted to say Three the word didn't come out». ${ }^{13}$ Podríamos deducir, por tanto, que el ideal de coalescencia propuesto por Cixous no se implementará hasta que se supere este sistema autoritario y arraigado.

Hasta ahora, hemos estado considerando la represión femenina que ejerce el sistema patriarcal, pero existe otra víctima menos evidente de esta poderosa fuerza social: el hombre. Cixous clarifica esta idea: «But he himself was buzzing, writhing in the web». ${ }^{14}$ La tela de araña podría considerarse como una metáfora que representa el sistema falocéntrico. Cixous elabora esta idea en su producción crítica, cuando afirma que:

"[p]hallocentrism is the enemy. Of everyone. Men's loss in phallocentrism is different from but as serious as women's. And it is time to change. To invent the other history». ${ }^{15}$

Estas palabras constituyen un grito de rebeldía, pues en ellas descubrimos que hay un espacio reservado para los hombres en el modelo de Cixous. Esta autora reconoce abiertamente que los varones resultan igualmente perjudicados al ver reducida la multiplicidad de su ser por el modelo patriarcal. Siempre que mantengan su lado femenino, existe una posibilidad de que puedan participar en la écriture féminine que Cixous propone.

12. Ibíd., p. 51.

13. Ibíd., p. 21.

14. Ibíd., p. 127.

15. Cixous, Hélène y Catherine ClÉMENT: op. cit., p. 83. 


\section{SUBVERSIÓN DE OPOSICIONES BINARIAS}

\subsection{Coalescencia de parejas opuestas}

En nuestra definición de "coalescencia» considerábamos la posibilidad de combinar dos o más elementos para constituir un todo distintivo, donde cada parte retiene al mismo tiempo su individualidad e igualdad con respecto a los otros miembros constituyentes. En esta sección, nos centramos en la primera de estas dos alternativas, es decir, la coalescencia de dos elementos. Cixous reacciona abiertamente contra el concepto patriarcal de oposiciones binarias que Conley clarifica:

"[Cixous] chooses not to take the route that denies sexual difference(s) but to accede to a play of differences that would not turn into oppositions $»^{16}$

De este modo, la coalescencia en Cixous sugiere la noción de "alteridad», definida por Claire Duchen como:

«another word for difference, implying radical 'otherness' as opposed to being different 'from' something else»"17

Al intentar eliminar esta oposición entre elementos binarios, Cixous sugiere lo que se ha denominado écriture féminine. Susan Sellers sugiere una definición de esta escritura que refleja con bastante precisión la conexión que surge entre elementos opuestos en este tipo de práctica:

«For Cixous, écriture féminine is the endeavour to write the other in ways which refuse to appropriate or annihilate the other's difference in order to create and glorify the self in a masculine position of mastery». ${ }^{18}$

La coalescencia de términos opuestos supone una cercanía entre ellos, pero siempre manteniendo una línea de separación con el fin de preservar la identidad de ambas partes. En el modelo de Cixous ambos sexos retienen sus rasgos idiosincrásicos, pero esto no les impide la coexistencia y el amor mutuo en la aceptación de sus diferencias dentro de un ámbito de igualdad. Así, Cixous concluye que «[t]he other must remain in all its extreme strangeness within the greatest possible proximity». ${ }^{19}$ Sin embargo, en este modelo de coalescencia, la autora no presentará los términos opuestos en una base de igualdad. Siguiendo el modelo desconstructivo de Derrida, Cixous ejecuta una inversión inicial de los valores patriarcales: donde el falocentrismo favorece aquellos términos asociados con la masculinidad, ella favorecerá los ligados a la feminidad. Trascien-

16. Conley, Verena Andermatt: Modern Cultural Theorists: Hélène Cixous, Toronto y Buffalo, University of Toronto Press, 1992, pp. 37-38.

17. DUCHEN, Claire: Op. cit., p. 49.

18. SelLeRS, Susan: Hélène Cixous: Authorship, Autobiography and Love, Cambridge (Inglaterra), Polity Press, 1996, pp. 11-12.

19. CIXOUS, Hélène: «Extreme Fidelity», en Susan Sellers (ed.): Writing Differences. Readings from the seminar of Hélène Cixous. (Trad. Ann Liddle y Susan Sellers), Milton Keynes, Open University Press, 1988 (1984), p. 29. 
de, no obstante, esta inversión simplista con una subversión evidente, donde visualiza un espacio para la coexistencia de ambos términos opuestos.

Tras matizar el uso de la coalescencia en Cixous, consideramos los dos tipos fundamentales que emplea en Angst: la coalescencia de parejas de términos opuestos y la coalescencia de múltiples conceptos. Comenzamos con el primer tipo, cuyo epítome radica en la fusión de sus escrituras teórica y creativa. Conley explica esta dualidad: "one has to go through theory to discover non-theory or poetry». ${ }^{20}$ Por esta razón, Angst no se puede denominar abiertamente "novela», puesto que en ella se funden los conceptos de escritura teórica y literaria. Para resaltar su modelo de coalescencia, Cixous recurre siempre a la siguiente estructura: en primer lugar, presenta los dos elementos que forman la coyuntura como mutuamente excluyentes, exponiendo el binarismo del patriarcado. A continuación, introduce la coalescencia de ambos términos, únicamente cuando los dos se conciben dentro de su modelo de escritura. Analicemos los modelos de coalescencia de parejas opuestas más significativos en Angst.

\subsubsection{Dentro/fuera}

La primera pareja de elementos opuestos es «dentro/fuera». Inicialmente se presentan como mutuamente excluyentes: "Turned inwards, inside, with no connection to an outside. Where the abyss was deepening». ${ }^{21}$ Sin embargo, cuando la autora considera su ámbito femenino, las dos realidades coexisten y retienen su carácter distintivo: "In and out of my body»; «it is another, still me, who is climbing outside me». ${ }^{22}$ En el primer ejemplo, la conjunción copulativa es significativa, pues indica una suma e implica que el segundo término puede existir a pesar de la existencia del primero. En el modelo patriarcal, «lo lógico» sería el uso de la conjunción disyuntiva $o$, que indica una elección excluyente de uno de los dos términos. En el segundo ejemplo, podemos deducir que el «I» o "self» está dentro mientras que "the other» ("another») está fuera, pero el hecho de que ambos sean equiparados ("still me») indica que las dos realidades coexisten dentro del mismo ser.

\subsubsection{Consciente/subconsciente}

Cixous propugna la coexistencia de estos dos términos puesto que, en el transcurso de su ficción, mezcla constantemente los ámbitos de la realidad y el sueño, en particular a través del uso metafórico del teléfono y el despertador como elementos disruptivos que transportan al individuo del mundo del sueño al mundo real: "Instantly the telephone rings. To bring you down. No need to pick up the receiver. You have already heard what death has to say to

20. Conley, Verena Andermatt: «Saying Yes to the Other», Dalhousie French Studies, 13 (1987), p. 94.

21. Cixous, Hélène: Angst, op. cit, p. 166.

22. Ibíd., pp. 7, 218. 
you». ${ }^{23} \mathrm{Al}$ conectar el teléfono con la muerte, la autora establece un paralelismo entre el orden simbólico-consciente y la muerte, de tal forma que favorece el inconsciente como el ámbito donde la feminidad reprimida puede florecer con libertad. Esta actitud positiva hacia el subconsciente se puede observar en la rebelión de la protagonista contra el teléfono (el patriarcado): «The telephone kept on ringing. It was over. From now on the best weapon was not to have one at all». ${ }^{24}$

\subsubsection{Tiempo real/tiempo subjetivo}

En estrecha conexión con la anterior, encontramos la dicotomía entre las concepciones del tiempo como presente/tiempo real y eternidad/tiempo subjetivo. Ambos extremos entran en contacto en la escritura ficcional de Cixous, puesto que referencias directas al tiempo real se combinan con las alusiones a una percepción subjetiva de la temporalidad. Por un lado, Cixous sigue una progresión lineal del tiempo al hacer alusión a distintas fechas: «5 June 1937»; 25 "January

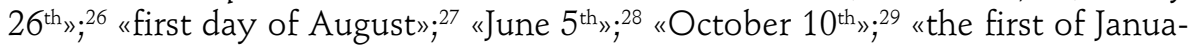
ry»; $; 0$ "September morning»; $; 1$ and "19 June, 1976». ${ }^{32}$ En la fecha final resulta evidente que ha transcurrido un período de tiempo de 39 años, una larga franja de tiempo para una ficción en la que el público lector tiene la impresión de que el tiempo no pasa. En realidad, éste es precisamente uno de los grandes logros de Cixous: una vez más, esta autora favorece uno de los términos opuestos, en este caso la percepción subjetiva del tiempo, la idea de que la maternidad (o feminidad en general) ha sido tradicionalmente asociada con un tiempo cíclico y eterno, frente a la linealidad del tiempo histórico, tradicionalmente asociado con la masculinidad. ${ }^{33}$

En el curso de la narrativa, la escritora alcanza esta impresión de una percepción subjetiva del tiempo a través de varios medios. Uno de ellos es la referencia a una inexistencia temporal, que se puede observar en afirmaciones como las siguientes: «it was always the same time»; ${ }^{34}$ "every second it is the same day beginning all over again». ${ }^{35}$ Esta impresión de estancamiento temporal se alcanza igualmente por medio de la repetición obsesiva de segmentos temporales,

23. Ibíd., p. 101.

24. Ibíd., p. 106.

25. Ibíd., p. 19.

26. Ibíd., pp. 26, 28.

27. Ibíd., p. 61.

28. Ibíd., p. 143.

29. Ibíd., p. 167.

30. Ibíd., p. 191.

31. Ibíd., p. 214.

32. Ibíd., p. 219.

33. Véase KrISTEVA, Julia: «Women's Time», en Toril Moi (ed.): The Kristeva Reader (Trad. Alice Jardine y Harry Blake), Oxford (Inglaterra), Basil Blackwell, 1986 (1979), pp. 187-213.

34. Cixous, Hélène: Angst, op. cit, p. 85.

35. Ibíd., p. 40. 
repetición que crea la sensación de que el tiempo es eterno: «a quarter of an hour»; ${ }^{36}$ "Ten years»; ${ }^{37}$ " five thousand years»; ${ }^{38}$ "between the $15^{\text {th }}$ and the $20^{\text {th }}$ » ${ }^{39}$ Por último, la preferencia de Cixous por una percepción temporal subjetiva resulta obvia cuando reduce el tiempo lineal a la noción de eternidad, haciendo que pasado, presente y futuro se fundan en un presente eterno:

"A minute ago in the room it was yesterday, and a new day every moment; in a minute, tomorrow, you get up again and again, and every minute is today.... Time in the room was not real time.... It was the Time of Times, eternity...». ${ }^{40}$

En cualquier caso, a pesar de la preferencia de Cixous por la eternidad, el tiempo real es igualmente necesario para establecer la coexistencia de términos binarios.

\subsubsection{Exilio/paraíso}

Una cuarta dicotomía es la de exilio/paraíso-hogar. Como es el caso de las parejas estudiadas previamente, estos dos términos coexisten en el modelo de Cixous:

"You can't say you're home, not really. It was so far away from me, in a remote time and space. And yet I was really home, in my mother's house to be precise». ${ }^{41}$

Además, como en los ejemplos precedentes, la autora favorece uno de los términos, en este caso, el exilio, puesto que este ámbito constituye la llave para entender la categoría del "otro", de lo diferente. Tenemos que distanciarnos de nosotros/as mismos/as (del «hogar») para comprender la diferencia (el exilio). Ésta es la razón por la que Cixous presenta a la protagonista de Angst como una doble marginada. En primer lugar, Cixous se refiere a la protagonista como un ser que habita "a foreign land». ${ }^{42}$ En segundo lugar, aparece como una extranjera con respecto a su propia identidad, puesto que su verdadera esencia ha sido reprimida por el orden simbólico-patriarcal: "My hands moved forward into the foreign flesh, of my sex». ${ }^{43}$

\subsubsection{Cuerpo/alma}

Otra categoría fundamental en la ficción de Cixous es la dicotomía entre cuerpolalma. Una vez más, la autora privilegia uno de los conceptos (el cuerpo). Este favoritismo se entiende porque las mujeres, debido a su función procreadora, han sido tradicionalmente consideradas como más cercanas al reino animal que el hombre, este último más próximo al mundo del espíritu y de la razón.

\footnotetext{
36. Ibíd., pp. 12-13, 17, 22, 26, 80.

37. Ibíd., pp. 111, 114, 119, 123.

38. Ibíd., pp. 113, 195.

39. Ibíd., pp. 39, 40.

40. Ibíd., p. 144.

41. Ibíd., p. 215.

42. Ibíd., pp. 65, 112.

43. Ibíd., pp. 180-181.
} 
La predisposición de Cixous hacia lo corporal no es gratuita; considerará este atributo como esencial y central a la feminidad y, por tanto, a su modelo de escritura femenina. En este sentido, Cixous concluye que «[b]y writing her self, woman will return to the body which has been more than confiscated from her». ${ }^{44}$ Esta posición especial que concede a lo corporal se puede observar en su presentación del cuerpo de la mujer como el de un animal a través de metáforas. $\mathrm{Su}$ objetivo es revalorar la naturaleza física atribuida a las mujeres frente a la trascendental atribuida a los hombres. De esta manera, la protagonista de Angst es identificada con «a dog»; 45 "the face of a bloodhound»; 46 « «ogs, rats and the offspring of mousers». ${ }^{47}$

A su vez, con el uso de la sinestesia Cixous contribuye a reforzar su idea de la coexistencia de elementos binarios. Esta figura literaria "where a stimulus applied to one sense involuntarily elicits a response from one or more others ${ }^{48}$ es extremadamente útil en la escritura de esta autora, puesto que la dota de un carácter tangible y "corporal» e ilustra la manera en que sentidos pertenecientes a distintos campos pueden coalescer. Los siguientes ejemplos de sinestesia en Angst muestran esta coalescencia de elementos aparentemente inconexos con gran precisión: "caressing it with your eyes»"49 (tacto y vista); "Milky voice of innocence $»^{50}$ (gusto y oído); "Voices hoarse with blood» ${ }^{51}$ (gusto y oído, si consideramos que la sangre está dentro de la boca y origina que la voz que se articula sea ronca). Como afirma Oboussier, el uso de la sinestesia «open[s] the way for new intersubjective economies and different ways of approaching the other». 52

\subsubsection{Masculinidad/feminidad}

La coalescencia de estos dos elementos conduce a la idea de bisexualidad defendida por Cixous, que define como "the location within oneself of the presence of both sexes, evident and insistent in different ways according to the individual, the nonexclusion of difference or of sex».53 Su defensa de la bisexualidad como el ámbito donde los roles sexuales confluyen se refleja en el curso de Angst, donde ambos términos opuestos coexisten. De esta manera, encontramos que términos como «man» o "he» se igualan al concepto de «mother» o se

44. CIXOus, Hélène: "The Laugh of the Medusa», en Elaine Marks e Isabelle de Courtivron (eds.): New French Feminisms. An Anthology (Trad. Keith Cohen y Paula Cohen), Sussex, The Harvester Press, 1981 (1975), p. 250.

45. CiXous, Hélène: Angst, op. cit, p. 44.

46. Ibíd., p. 48.

47. Ibíd., p. 52.

48. OвоUSSIER, Claire: «Synaesthesia in Cixous and Barthes», en Judith Still (ed.): Women and Representation, London, WIF, 1995, p. 115.

49. Cixous, Hélène: Angst, op. cit, p. 48.

50. Ibíd., p. 59.

51. Ibíd., p. 129.

52. OвOUSSIER, Claire: op. cit., p. 131.

53. CiXous, Hélène y Catherine CLÉMENT: op. cit., p. 85. 
asocian con partes distintivas del cuerpo femenino, como «womb» 0 «breasts». ${ }^{54}$ La figura hermafrodita del «wolf-dragon» aparece descrita como "half-female», "half-bird» (de nuevo la conexión del cuerpo femenino con el reino animal), "like a man", mostrando al mismo tiempo ambos órganos sexuales. ${ }^{55}$

La fluctuación entre «two principal libidinal economies» (masculina y femenina) que, según Cixous, conduce a la bisexualidad, es reconocida por Sellers en su introducción a The Hélène Cixous Reader, donde, haciéndose eco de Cixous concluye:

"we all continually fluctuate between gender roles, sometimes assuming defensive, 'masculine' positions, at other times willing to risk prohibition, and at other times combining elements of each». ${ }^{56}$

Esta bisexualidad aparece también reflejada en Angst, donde la protagonista fluctúa entre el papel defensivo masculino cuando se aferra a la seguridad proporcionada por el interior ${ }^{57} \mathrm{y}$ el rol arriesgado femenino ( $\mathrm{I}$ am told it is too cold there for human beings -never mind-you chance it -here in this camp there's no love» $\left.{ }^{58}\right)$.

Sin embargo, la coalescencia de las identidades masculina y femenina dentro de un mismo ser se muestra con mayor claridad en la frecuente identificación del yo narrativo (un yo femenino) con su homólogo masculino, representado por el pronombre personal he: "Talking to me all the while as if he were having a conversation with himself; with his body». ${ }^{59}$ Más frecuentemente, el pronombre masculino en tercera persona es reemplazado por una segunda persona, cuando el yo narrativo se dirige directamente a él (al homólogo masculino de la protagonista). En este caso, la coalescencia de ambos se retiene: "And then, if you want to hurt me, don't. I don't want you to hurt yourself». ${ }^{60}$ Aludiendo a Tristán e Isolda, el narrador los califica como:

"[s]eparate, different, one body seemed slightly more feminine, perhaps the other body more masculine, but I wasn't sure about anything, a feminine man, a woman-life personified». ${ }^{61}$

En este caso, más que coalescencia, encontramos un ejemplo de fusión de términos. El resultado de la mezcla de las economías libidinales masculina y femenina parece inclinarse favorablemente hacia el lado femenino; la idea del "hombre femenino", donde encontraríamos coalescencia, es modificada por la referencia de la autora a una «mujer», lo que ilustra la preferencia de Cixous por esta figura. En este sentido, la autora no sólo favorece la feminidad, sino que

54. Cixous, Hélène: Angst, op. cit, pp. 19, 38, 44, 57, 61, 68, 77, 79, 89, 189.

55. Ibíd., pp. 74, 78, 88.

56. Sellers, Susan (ed.): The Hélène Cixous Reader, Londres, Routledge, 1994, p.xxviii.

57. Cixous, Hélène: Angst, op. cit, pp. 9, 19, 23, 167.

58. Ibíd., p. 41.

59. Ibíd., p. 161.

60. Ibíd., p. 156.

61. Ibíd., p. 100. 
presenta al yo narrativo como una mujer: «I» como una "Woman»;62 «I» como "a mother»; 63 "I» o el genérico "one» equiparados con pronombres femeninos como el personal «she» o el reflexivo "herself». ${ }^{64}$

Cixous privilegia el cuerpo de la mujer por dos razones principales: por un lado, para corregir la posición tradicional de las mujeres como seres marginales dentro de una sociedad patriarcal; por otro, para llamar la atención sobre la diferencia sexual que las dota de un carácter distintivo y único en lo que se refiere a la maternidad, un estado del que los hombres son excluidos. Esta posibilidad es esencial para entender la práctica de la coalescencia, puesto que la maternidad es el prototipo de dicha unión de elementos opuestos, como la propia Cixous explica:

"How could the woman, who has experienced the not-me within me [i.e. embarazo], not have a particular relationship to the written? To writing as giving itself away (cutting itself off) from the source? $)^{65}$

La subjetividad femenina, debido al acceso de las mujeres a la maternidad, es una subjetividad que "splits apart without regret»" ${ }^{66}$ frente al "[m]asculine narcissism and male need for recognition». ${ }^{67}$

Sin embargo, a pesar de su interés por el cuerpo de la mujer, la autora no excluye a los hombres de su propuesta. Al final de Angst, Cixous deja lugar para la coalescencia en el hombre: «He was a man heavy as death, light as death, who smiled to himself. He had shrunk to normal size». ${ }^{68} \mathrm{La}$ coalescencia de términos opuestos en él se puede observar en la unión de "heavy» y "light». Esto es posible porque este hombre (y en teoría cualquier hombre) ha abandonado su posición jerárquicamente superior para aceptar la diferencia existente en elementos considerados «inferiores», una diferencia importante dentro de un cosmos en el que todos los términos son iguales, independientemente de sus diferencias $y$, por tanto, elementos integradores de un todo. En este sentido, el cambio de letra mayúscula en "God himself»" ${ }^{69}$ a minúscula en «he, god» ${ }^{70}$ indica el final de la verdad única y totalitaria del patriarcado, y el principio de un universo plural, donde las diferencias coexisten y donde la verdad masculina es simplemente una más dentro de un espectro de verdades potenciales. Al mostrar la compatibilidad del hombre con la nueva forma de escritura que Cixous propone, esta autora prueba que, aunque "[she] remains loyal to women's causes her more general interest is in the constructions and motivations of the human subject». ${ }^{71}$

62. Ibíd., pp. 33-4, 40, 44-5, 48, 64, 214.

63. Ibíd., pp. 46, 89.

64. Ibíd., pp. 42, 70, 119.

65. CiXous, Hélène y Catherine ClÉMENT: Op. cit., p. 90.

66. CiXOus, Hélène: Angst, op. cit, p. 26.

67. CONLey, Verena Andermatt: Modern Cultural Theorists, op. cit., p. 31.

68. Cixous, Hélène: Angst, op. cit, p. 216.

69. Ibíd., p. 209.

70. Ibíd., p. 216.

71. Sellers, Susan: Hélène Cixous: Authorship, Autobiography and Love, op. cit., p. 3. 
Es en este punto donde la coalescencia de las economías masculina y femenina se observa con mayor claridad.

\subsection{Coalescencia de múltiples componentes}

\subsection{1. «Why-Nots»}

En cuanto a la coalescencia de más de dos componentes que, en su unión, dan lugar a un todo unitario, nuestro primer objeto de análisis es lo que denominamos, citando a Cixous, "why-nots»: todas aquellas posibilidades que no llegan a materializarse pero que son partes constitutivas de la identidad del individuo. Cixous asegura que:

"We are possible. We only need to avoid closing up the parentheses in which our 'why-nots' live.... However and by chance I am a woman, and I belong to the human race». ${ }^{72}$

Con esta aseveración, la autora nos incita a ser tolerantes y aceptar todo aquello que difiere de nosotros/as. Todas esas posibilidades se fundirán en un macrocosmos de multiplicidad. Como Conley afirma: «Pluralism replaces binaries». ${ }^{73}$ En Angst, Cixous alude con frecuencia a un haz infinito de posibilidades, todas ellas igualmente aceptables, incluso cuando algunas puedan parecer completamente inalcanzables (como es su noción de la concepción humana, no en la matriz de la mujer, sino en los pulmones ${ }^{74}$ ). Este pluralismo de posibilidades queda aún más claramente reflejado en los siguientes ejemplos extraidos de su narrativa:

«Two or three, four already. Perhaps six. Possibly Saturday? Very possible. Monday for certain. It could be any day... let two be three. And it was so. Why can't you really feel that two is six?» (Angst 41-2); «Within me were all the selves I would become...». ${ }^{75}$

\subsubsection{El sujeto: coalescencia de múltiples identidades}

Relacionada con esta lista interminable de posibilidades, Cixous concibe su noción del «sujeto». Según explicamos previamente, esta autora parte del sujeto femenino como el centro de su propuesta, hasta tal punto que, en su definición del sujeto, asegura que:

"It is not 'I am a woman', full stop.... Woman is she who 'is' woman and, woman making her way forward in the world taking heed ${ }^{76}$

Siguiendo a Cixous, Oboussier también concluye que el espacio femenino es un "espacio intersubjectivo». ${ }^{77}$ Por tanto, Cixous aplica esta intersubjetividad

72. Cixous, Hélène: «Extreme Fidelity», op. cit., p. 34.

73. CONLEY, Verena Andermatt: Modern Cultural Theorists, op. cit., p. 90.

74. Cixous, Hélène: Angst, op. cit., p. 21.

75. Ibíd., p. 46.

76. CIXOUS, Hélène: «Extreme Fidelity», op. cit., p. 28.

77. OBOUSSIER, Claire: op. cit., p. 127. 
a su voz narrativa que se convierte en un "plural narrator divided into hundreds of subjects, without limit between one sex and the other (ne-uter)». ${ }^{78}$ Esta pluralidad narrativa se refleja en Angst, donde a la narradora no se le atribuye una identidad totalitaria y unificada, sino fragmentada y compuesta por numerosas parcelas. Dicha multiplicidad se manifiesta en los siguientes casos: «blood running out of all my mouths»; $; 9$ "in the stories of my lives»; $; 0$ "I will show you all the beings I have been.' Countless selves». ${ }^{81}$

Otra forma efectiva por la que Cixous introduce la multiplicidad de la persona narrativa es el juego con los pronombres personales. I, You (con una referencia en singular), he or she (todos ellos aludiendo a la voz narrativa) se equiparan al pronombre personal en plural we, que funciona como el «tercer término» necesario para solventar la separación existente entre términos opuestos. Al observar este juego constante con los pronombres, el público lector tiene la impresión de que la identidad de la voz narrativa no es fija, sino múltiple e indescifrable: "to you; to me... or one of us»; "he is me is us-we»; "I am hurting us». ${ }^{82}$

Este cambio constante en la identidad de los pronombres personales también constituye una técnica muy útil para presentar al yo ficcional como un todo unitario, compuesto de tres partes constitutivas, a saber: la voz narrativa, la protagonista y la autora de la ficción (o sea, Hélène Cixous). En el transcurso de la narrativa, nos encontramos con un frecuente desdoblamiento de la voz narrativa en los pronombres $I$ y you ${ }^{83}$ refiriéndose indistintamente a la narradora y a la protagonista. Aunque se trata de la misma persona, por medio de esta técnica Cixous atrae nuestra atención hacia la multiplicidad del sujeto, que aparece aún más fragmentado cuando, al final de la novela, nos percatamos de que la propia autora es la narradora y la protagonista de esta ficción: "An hour ago I existed, I believed in myself, not a moment ago I was called Hélène-you will never believe it again». ${ }^{84}$ Una vez más, la idea de un tercer término aparece, un elemento que establece la conexión necesaria entre los dos términos previamente analizados.

Por último, Cixous ilustra la multiplicidad del sujeto a través de la multiplicidad de voces que se funden (otro ejemplo de coalescencia) en la voz narrativa. De este modo, Conley opina que "the artist's work is not creation ex nihilo, but is elaborated as an invention with others, through a fraying of voices». ${ }^{85}$ Con frecuencia, Cixous emplea voces directamente tomadas de las identidades más representativas del sistema falocéntrico, pero su objetivo es paródico, usando

78. CONLEY, Verena Andermatt: Modern Cultural Theorists, op. cit., p. 23.

79. CiXous, Hélène: Angst, op. cit., p. 53.

80. Ibíd., p. 70.

81. Ibíd., p. 142.

82. Ibíd., pp. $27,43$.

83. Ibíd., pp. 36, 59, 159, 161.

84. Ibíd., p. 210.

85. CONLEY, Verena Andermatt: Modern Cultural Theorists, op. cit., p. 4. 
dichas voces como el punto de partida para contradecir las ideas del patriarcado. Por ejemplo, Cixous cita la Biblia varias veces. En una de ellas, hace referencia a uno de los diez mandamientos: "Thou shalt not commit adultery». ${ }^{86} \mathrm{Su}$ intención es reaccionar contra el modelo monogámico impuesto por el patriarcado a través de su texto más significativo, la Biblia. La protagonista de Angst dice: "I would have committed adultery, if only the guilt would go away». ${ }^{87} \mathrm{Se}$ alza contra el sistema monogámico dictado por el orden simbólico para la preservación del poder del hombre. Además de las alusiones bíblicas, Cixous emplea referencias a otras figuras de renombre dentro del orden falocéntrico, figuras masculinas tan adoradas y reconocidas como Donne, Kafka o Saroyan, ${ }^{88}$ todos ellos asociados con un modelo que liga a las mujeres con la idea de muerte. Además, Cixous hace uso de muchas otras voces filtradas en último término por la voz narrativa, como "Guilt»; "Man with smile» (que representa al patriarcado) $;{ }^{90}$ "Amnesia» ${ }^{91}$ su «Grand-mother ${ }^{92} \mathrm{y}$ "the Pangs of Anguish». ${ }^{93}$

\subsubsection{Multiplicidad en el lenguaje}

La coalescencia en Cixous de múltiples componentes se observa con notable claridad en su propuesta de un nuevo lenguaje, donde reacciona contra las limitaciones impuestas por el vehículo de comunicación patriarcal. En este sentido, considera que "language conceals an invincible adversary, because it's the language of men and their grammar». ${ }^{94}$ Debido a esta desventaja para las mujeres, la autora añade que:

"If woman has always functioned 'within' the discourse of man... it is time for her to dislocate this 'within', to explode it, turn it around and seize it; to make it hers, containing it, taking it in her own mouth, biting that tongue with her very own teeth to invent for herself a language to get inside of $\gg .{ }^{95}$

Para alcanzar este objetivo, Cixous comienza por ilustrar la opresión del lenguaje; cómo éste conduce a la falta de representación de las mujeres y a su muerte simbólica. La autora dibuja esta imagen en Angst: «by accident, you read the last page of the Book of the Dead and find your own name in it!».96

Resulta significativo que, cuando Cixous intenta reflejar las limitaciones impuestas por el lenguaje, escoge nombres propios como el epítome de dicha represión. Así, concibe los nombres como "social tools, rigid concepts, little

86. Cixous, Hélène: Angst, op. cit., p. 34.

87. Ibíd., p. 34.

88. Ibíd., pp. 113, 143, 155.

89. Ibíd., p. 38.

90. Ibíd., pp. 59, 76.

91. Ibíd., pp. 74-75.

92. Ibíd., p. 84.

93. Ibíd., pp. 92-93.

94. CIXOUS, Hélène: "The Laugh of the Medusa», op. cit., p. 257.

95. Cixous, Hélène: Angst, op. cit., p. 189.

96. Ibíd., p. 67. 
cages of meaning assigned ... to keep us from getting mixed up with each other, without which the Society of Cacapitalist Siphoning would collapse». ${ }^{97}$ $\mathrm{Su}$ conclusión es que "[n]ames must be suppressed». ${ }^{98}$ En el nuevo lenguaje que sugiere, Cixous recurre a interminables variaciones léxicas que aluden a múltiples facetas de un mismo individuo, escapando así de cualquier reducción simplista de potencialidades. El ejemplo más obvio lo constituye la identidad de la voz narrativa. A lo largo de la ficción, la protagonista es aludida por medio de numerosas variaciones léxicas: "She-who-knows-who-I-am»; "She-who-ismy-Answer»; 100 "She-who-is-waiting-for-you»; ${ }^{101} \mathrm{o}$ "She-whom-he-is-waitingfor». ${ }^{102}$ Tan sólo al final el nombre propio de Hélène Cixous es mencionado, pero únicamente para aludir a la muerte causada por las limitaciones de las etiquetas léxicas y del lenguaje restrictivo patriarcal: «not a moment ago I was called Hélène-you will never believe it ever again». ${ }^{103}$ De este modo, la etiqueta restrictiva de Hélène contrasta con la multiplicidad de etiquetas léxicas que, como Hélène, aluden a la misma persona, pero que, al contrario que el nombre propio, reflejan la multiplicidad de las diferentes facetas del mismo individuo que coalescen para formar el mismo todo unitario. Un efecto similar se consigue por medio del uso de iniciales que, al contrario que los nombres completos, nos permiten imaginar un universo interminable de posibles identidades: "S», «V», «F».104

Finalmente, Cixous consigue su ideal de multiplicidad en el lenguaje por medio del uso abundante de metáforas. Utiliza alusiones metafóricas, en primer lugar para atribuir a los términos abstractos la materialidad que su escritura corporal requiere; en segundo lugar, para materializar la coalescencia de múltiples posibilidades. Enumeremos los casos más representativos de metáfora en este sentido: «the corridors of my dream»; ${ }^{105}$ "too many keys»; ${ }^{106}$ los sueños como "passageways»; ${ }^{107}$ "The stairs were endless»; $;^{108}$ "ways», "crossroads». ${ }^{109}$

Aunque el modelo de Cixous parece muy comprensivo, una panacea para la enfermedad patriarcal, precisamente debido a esta dimensión idealista, ha recibido numerosas críticas por su carácter utópico. Al menos, podemos concluir que, incluso cuando marcada por esta utopía, la propuesta de coalescencia de Cixous

97. Cixous, Hélène: «Coming to Writing», en Deborah Jenson (ed.): "Coming to Writing» and Other Essays: Hélène Cixous (Trads. Sarah Cornell, Deborah Jenson, Ann Liddle y Susan Sellers), Cambridge (Massachusetts), Harvard University Press, p. 91.

98. CiXOUS, Hélène: Angst, op. cit., p. 48.

99. Ibíd., p. 14.

100. Ibíd., p. 15.

101. Ibíd., p. 29.

102. Ibíd., p. 58.

103. Ibíd., p. 210.

104. Ibíd., p. 130.

105. Ibíd., p. 10.

106. Ibíd., p. 49.

107. Ibíd., p. 90.

108. Ibíd., p. 109.

109. Ibíd., pp. 134-135. 
Coalescencia: una aproximación a la escritura de Hélène Cixous

puede ser muy efectiva para persuadir al público lector de ser más tolerante en cuanto a las diferencias individuales. Aunque su modelo es idealista, constituye una forma de escribir sobre el tema de la "diferencia» sin pensar en términos del poder autoritario sobre los demás, deshaciendo de este modo el lazo que tradicionalmente ha existido entre los conceptos de "diferencia» $\mathrm{y}$ «dominio». 